\title{
Spotted Fever Group Rickettsioses in Israel, 2010-2019
}

\author{
Regev Cohen, Talya Finn, Frida Babushkin, Yael Paran, Ronen Ben Ami, Alaa Atamna, \\ Sharon Reisfeld, Gabriel Weber, Neta Petersiel, Hiba Zayyad, Eyal Leshem, Miriam Weinberger, \\ Yasmin Maor, Nicola Makhoul, Lior Nesher, Galia Zaide, Dar Klein, Adi Beth-Din, Yafit Atiya-Nasagi
}

In a multicenter, nationwide, retrospective study of patients hospitalized with spotted fever group rickettsiosis in Israel during 2010-2019, we identified 42 cases, of which 36 were autochthonous. The most prevalent species was the Rickettsia conorii Israeli tick typhus strain (n $=33,79 \%$ ); infection with this species necessitated intensive care for $52 \%$ of patients and was associated with a $30 \%$ fatality rate. A history of tick bite was rare, found for only $5 \%$ of patients; eschar was found in $12 \%$; and leukocytosis was more common than leukopenia. Most (72\%) patients resided along the Mediterranean shoreline. For 3 patients, a new Rickettsia variant was identified and had been acquired in eastern, mountainous parts of Israel. One patient had prolonged fever before admission and clinical signs resembling tickborne lymphadenopathy. Our findings suggest that a broad range of Rickettsia species cause spotted fever group rickettsiosis in Israel.

Cpotted fever group rickettsioses (SFGRs) are ar$\checkmark$ thropodborne diseases caused by obligate intracellular, gram-negative bacteria of the genus Rickettsia.

Author affiliations: Laniado Hospital, Netanya, Israel (R. Cohen,

T. Finn, F. Babushkin); Technion-Israel Institute of Technology, Haifa, Israel (R. Cohen, T. Finn, S. Reisfeld, G. Weber); Tel-Aviv Sourasky Medical Centre, Tel-Aviv, Israel (Y. Paran, R. Ben Ami); Tel Aviv University, Tel Aviv (Y. Paran, R. Ben Ami, A. Atamna, E. Leshem, M. Weinberger, Y. Maor); Rabin Medical Centre, Petah-Tikva, Israel (A. Atamna); Hillel Yaffe Medical Centre, Hadera, Israel (S. Reisfeld); Carmel Medical Centre, Haifa (G. Weber); Rambam Medical Centre, Haifa (N. Petersiel); Padeh Poriya Medical Centre, Tiberias, Israel (H. Zayyad); Bar Ilan University, Safed, Israel (H. Zayyad,

N. Makhoul); Sheba Medical Centre, Ramat Gan, Israel (E. Leshem); Shamir (formerly Assaf Harofeh) Medical Center, Zerifin, Israel (M. Weinberger); Edith Wolfson Medical Centre, Holon, Israel (Y. Maor); Galilee Medical Centre, Nahariya, Israel (N. Makhoul); Soroka Medical Centre, Beer Sheba, Israel (L. Nesher); Ben-Gurion University of the Negev, Beer Sheba (L. Nesher); Israel Institute for Biological Research, Ness Ziona, Israel (G. Zaide, D. Klein,

A. Beth-Din, Y. Atiya-Nasagi)

DOI: https://doi.org/10.3201/eid2708.203661
SFGRs are associated with $\approx 20$ species of Rickettsia, of which 16 are considered human pathogens $(1,2)$. Recent introduction of molecular methods provided more information about SFGR agents causing human disease and enabled their identification, for which the clinical significance of some remains lacking (3). $R$. conorii complex, the etiologic agent of Mediterranean spotted fever, includes 4 strains: $R$. conorii Malish (cause of Mediterranean spotted fever), $R$. conorii Astrakhan (cause of Astrakhan fever), R. conorii Indian tick typhus (cause of Indian tick typhus), and $R$. conorii Israeli tick typhus strain (ITTS, cause of Israeli spotted fever [ISF]) (4-7).

ISF begins as fever followed by a maculopapular rash, usually involving the palms and soles and frequently accompanied by systemic symptoms. Most cases are self-limiting, but some may lead to organ failure and death. Clinical and epidemiologic presentations caused by other strains of rickettsiae may vary $(5,8-11)$. Studies from Portugal indicate that compared with Mediterranean spotted fever, ISF is characterized by lower rates of eschar and tick-exposure history, higher frequency of gastrointestinal symptoms, and greater severity of illness with a high case-fatality rate $(10,12,13)$. Case-fatality rates in Israel before 1998 were reportedly $0.7 \%$, but incidence in some years (e.g., 1997) was higher (3.5\%) (14). Since 1998, several case reports of fatal SFGR in Israel have been published $(9,15-17)$, along with reports of 22 other patients with sepsis requiring hospitalization $(9,17-22)$. Of these, isolates from only 3 patients were sequenced and identified as $R$. conorii ITTS; 2 of these patients exhibited purpura fulminans and all 3 died $(9,15)$. Few studies of ISF have been conducted in Israel; most were conducted during the 1990s and relied on serologic diagnostic methods that cannot differentiate between the SFGRs (23). In Israel, SFGR is a notifiable disease, and in recent years, incidence has increased; 51 cases (including 7 deaths) were reported to the Israeli Ministry of Health in 2017, compared 
with the average yearly incidence of 26 cases during 2014-2016 (24).

The etiology of SFGR in Israel is thought to be $R$. conorii ITTS on the basis of limited molecular identification of this strain from clinical cases and from ticks $(9,15,25)$. However, the yearly variations in disease severity and clinical manifestations are intriguing and may suggest involvement of $>1$ species of spotted fever group (SFG) Rickettsia. Using a large database from the national reference center, we studied the specific species of Rickettsia that cause SFGRs in Israel and characterized their unique epidemiology and clinical features. Institutional review board approval was granted at the principal investigator site (0002-19-LND) and for each participating institute.

\section{Methods}

\section{Study Design}

We conducted a multicenter, retrospective study of hospitalized patients with an SFGR during 20102019. The study included SFGR diagnosed by molecular methods at the Israel Institute for Biological Research (IIBR; Ness Ziona, Israel), which serves as the national reference center for Rickettsia. Blood and tissue samples from hospitalized patients with a suspected SFGR are occasionally submitted to the IIBR for molecular diagnosis, at the discretion of the treating physician. For cases of successful molecular identification, the referring medical center was requested to provide patient demographic and clinical data from the medical charts at each participating site. Deidentified data were integrated into a central database.

\section{Serology and Molecular Diagnoses}

The IIBR tested serum samples for antibodies against $R$. conorii and $R$. typhi by an in-house immunofluorescence assay (cutoff for IgG of 1:100), as previously described (26). Skin biopsy samples, whole blood, cerebrospinal fluid, and other tissues were tested by PCR $(17,27)$. Stored SFG-positive Rickettsia DNA samples and sequenced unique regions from 4 conserved Rickettsia genes were batch tested (primers listed in Table 1). R. africae was identified by real-time PCR targeting an internal transcribed spacer (30). These analyses enabled identification of ISF, R. africae, R. conorii Malish strain, and a new Rickettsia variant. Each set of reactions included a positive control and nontemplate as a negative control. The same primers were used for sequencing as for amplification. For species-level identification, we compared sequence results by using BLAST (http:/ / www.ncbi.nlm.nih.gov/BLAST).

\section{Mapping Locations of SFGR Acquisition}

We recorded the site of presumed rickettsiosis acquisition for autochthonous cases. When the site was unknown, we used the patient's address.

\section{Statistical Analyses}

We used descriptive statistics to summarize patient characteristics. We calculated differences between categorical and continuous variables by using Pearson $\chi^{2}$, Student $t$, and Mann-Whitney tests, as appropriate. We used 1-way analysis of variance to analyze differences among groups. For statistical analyses, we used SPSS Statistics 25 (https://www.ibm.com). We considered 2 -sided $p<0.05$ to be significant.

\begin{tabular}{|c|c|c|}
\hline Primer & Target gene & Primer sequence, $5^{\prime} \rightarrow 3^{\prime}$ \\
\hline $213 \mathrm{~F}$ & OmpA & AATCAATATTGGAGCCGGTAA \\
\hline 667R & OmpA & ATTTGCATCAATCGTATAAGTAGC \\
\hline $120 \mathrm{~F}$ & OmpA & AAGGAGCTATAGCAAACGGCA \\
\hline $760 R$ & OmpA & TATCAGGGTCTATATTCGCACCTA \\
\hline 760newF & OmpA & TAGGTGCGAATATAGACCCTGATA \\
\hline 1231R & OmpA & TGGCAATAGTTACATTTCCTGCAC \\
\hline $373 F$ & gltA & TTGTAGCTCTTCTCATCCTATGGC \\
\hline 1138R & gltA & CATTTGCGACGGTATACCCATA \\
\hline Rico173F & gltA & CGACCCGGGTTTTATGTCTA \\
\hline $1179 \mathrm{R}$ & gltA & TCCAGCCTACGATTCTTGCTA \\
\hline gltA_EXT_R & gltA & TACTCTCTATGTACATAACCGGTG \\
\hline gltA_NES_F & gltA & ATGATTGCTAAGATACCTACCATC \\
\hline 1497_R & OmpB & CCTATATCGCCGGTAATT \\
\hline 3462_F & $O m p B$ & CCACAGGAACTACAACCATT \\
\hline 4346_R & $O m p B$ & CGAAGAAGTAACGCTGACTT \\
\hline 607 F & OmpB & AATATCGGTGACGGTCAAGG \\
\hline D1390R & Sca4 & CTTGCTTTTCAGCAATATCAC \\
\hline D767F & Sca4 & CGATGGTAGCATTAAAAGCT \\
\hline
\end{tabular}




\section{Results}

\section{SFGR Cases Diagnosed by IIBR in the Past Decade}

In the 10-year study period, 1,985 cases of rickettsioses from the community and hospitals countrywide were diagnosed at IIBR by serologic testing; 811 were SFGR and 1,174 were murine typhus. Another 89 cases were positive by PCR, 66 for SFGR and 23 for murine typhus (Figure 1, panels A, B). Comparing 2010-2014 with 2015-2019 indicated an increased number of serologic tests performed yearly (from an average of $\approx 2,000$ to $\approx 6,500$ ). The percentage of positive serologic results decreased from 3\% in 2010-2013 to $1.2 \%$ in $2014-2016$ and then increased again to $2 \%$ in 2017-2019. Concomitantly, a 4-fold increase in total PCR tests performed was accompanied by a 7-fold increase in positive PCR results (average of 2.2-15.6 positive cases/year in the 2 periods). Of the 66 SFGR cases positive by PCR, $42(64 \%)$ were identified to the strain level; for the other 24 cases, the classification failed, probably because of a low number of DNA copies in the original sample.

\section{Rickettsia Strains}

The most prevalent Rickettsia strain in this study was $R$. conorii ITTS, found in 33/42 (79\%) of cases identified to the strain level. The other strains accounted for 4 cases of $R$. africae infection, 2 of $R$. conorii Malish strain infection, and 3 of the newly identified Rickettsia variant (27). Most (36/42, 86\%) cases were autochthonous: $32 / 36$ (89\%) were caused by R. conorii ITTS, 1 by $R$. conorii Malish strain, and the remaining 3 by the new Rickettsia variant (Figure 1, panel B). The 6 imported cases included 4 infections with $R$. africae, all acquired during a safari (in South Africa, Mozambique, Zimbabwe, or Botswana); 1 R. conorii ITTS imported from Cyprus; and 1 R. conorii Malish strain imported from New Delhi, India.
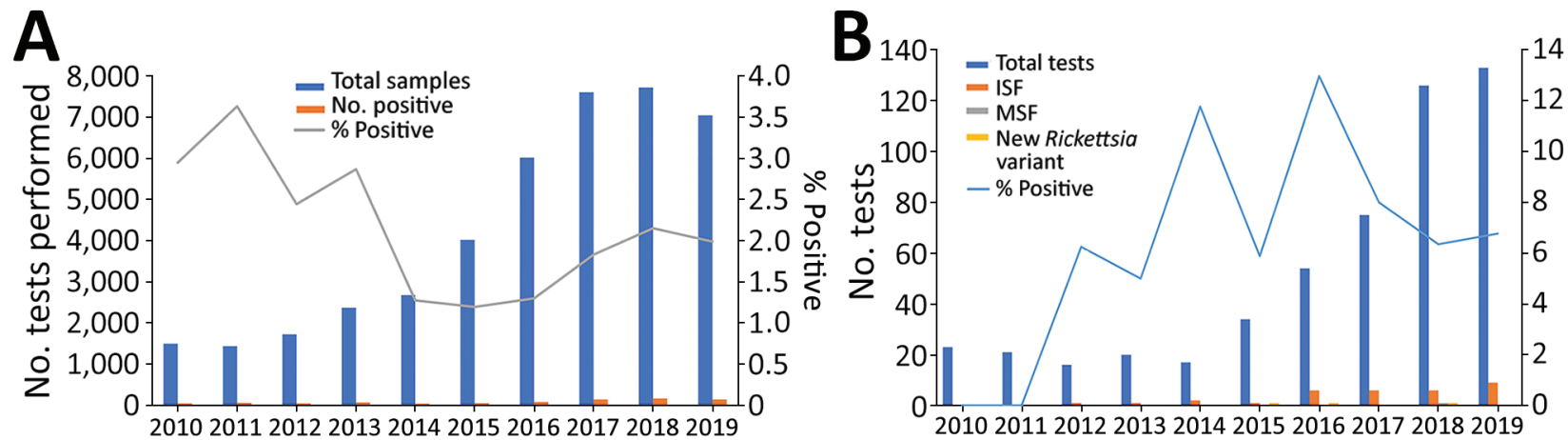

Figure 1. Spotted fever group rickettsioses, Israel, 2010-2019. A) Serologic tests performed in the Israeli central laboratory for rickettsiosis and number of positive cases per year. B) Autochthonous cases identified to the strain level. ISF, Israeli spotted fever; MSF, Mediterranean spotted fever.
Molecular diagnosis was performed from skin biopsy samples from 27 patients (19 with ISF, 3 with new Rickettsia variant infection, 1 with $R$. conorii Malish strain infection, and 4 with African tick bite fever [ATBF]); from the blood of 19 patients (16 with ISF, 2 with R. conorii Malish strain, and 1 with new Rickettsiae variant); and from cerebrospinal fluid, lymph node, and liver biopsy postmortem samples from 1 patient with ISF. Seven patients were positive by PCR of samples from $>1$ source, mostly skin biopsy and whole blood. Serologic testing was performed for $36 / 42(86 \%)$ patients; results were available for 26 . Of these, results were negative for $16(61 \%)$ and the median time between disease onset to last serology test was 6.2 days (range $0-11$ days); for 10 (39\%), the result was either positive ( 5 patients) or borderline (5 patients) and median time from disease onset to last serologic test 15.5 days (range $2-29$ days).

\section{Geographic and Seasonal Distribution of Cases}

The 36 autochthonous cases were reported from 12 hospitals representing most areas of Israel: 6 from central Israel, 4 from northern Israel, and 2 from southern Israel. $R$. conorii ITTS was reported from all but 1 hospital. All cases of $R$. africae infection were reported from 1 hospital. The most abundant concentration of cases (26/36, 72\%, all R. conorii ITTS) was along the Mediterranean shoreline, with the highest aggregation of cases in the Sharon and Haifa districts (Figure 2, panel A). Ten cases were acquired inland, of which 6 were caused by R. conorii ITTS and 3 the new Rickettsia variant. Those 3 cases were presumably acquired in more mountainous areas of Israel. The $R$. conorii Malish strain was acquired in the desert region near Beer-Sheba.

Nearly half $(27 / 61,44 \%)$ of SFGR cases occurred during the 3 summer months, peaking in August. For the other seasons, $24 \%$ were recorded in the fall, $24 \%$ in spring, and $8 \%$ in winter. 


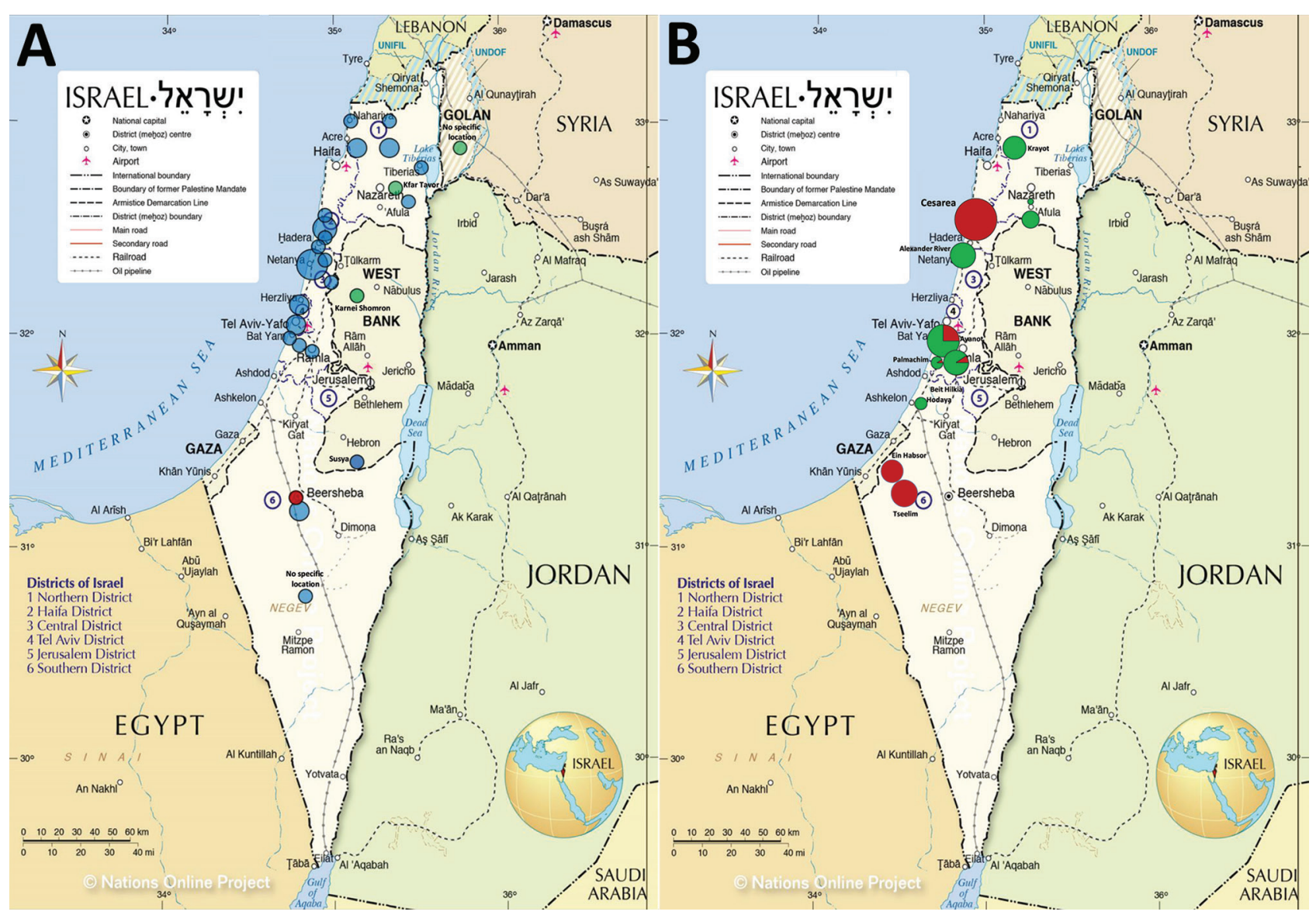

Figure 2. Geography of spotted fever group rickettsioses, Israel, 2010-2019. A) Presumed areas of autochthonous infection acquisition ( $n=36$ cases). B) Tick collection sites and tick species collected during 2014 by Rose et al. (31). ISF, Israeli spotted fever; MSF, Mediterranean spotted fever. Source: Nations Online Project (https://www.nationsonline.org).

\section{Patient Characteristics and Demographics}

Median patient age was 50.5 years (interquartile range [IQR] 26-66 years), 25/42 (60\%) were male, and $21 / 42(50 \%)$ had $\geq 1$ significant previous medical conditions (diabetes mellitus and dyslipidemia were the most prevalent, at 19\% each) (Table 2). We found no significant differences between the 4 groups of rickettsial infections with regard to patient age, sex, and previous medical conditions. Although a history of tick exposure was rarely reported $(2 / 42,5 \%$ of patients, all in the ISF group), domestic animal exposure was quite common (reported by $25 / 42,60 \%$ of patients), most commonly to dogs (20/42, $48 \%)$ and cats $(5 / 42,12 \%)$. Few patients reported exposure to cattle, sheep, or rats.

\section{Clinical Features}

All patients were hospitalized except for 1 with ATBF, who had mild disease (Table 3). The mean duration of stay was 11.9 days, and median (IQR) was 5 (3-10) days. Fever affected 41/42 (98\%) patients. The mean number of days with fever until hospitalization was 5.2 , and the median (IQR) was 5 (3-6) days; $98 \%$ of patients were hospitalized by day 9 of fever onset. For patients infected with the new Rickettsia variant, the mean interval was significantly longer $(13.6 \pm 13.3$ days, range 5-29 days; $p=0.002$ when compared with the other groups); 1 patient in this group had fever of unknown origin for 29 days before hospitalization.

Skin involvement in SFGR patients - either rash or eschar-was nearly universal $(41 / 42,98 \%)$. Systemic rash was documented for $37 / 42(88 \%)$ of patients and absent for all 4 with ATBF, 1 with $R$. conorii Malish strain infection. The prevalent rash type was maculopapular $(22 / 42,53 \%)$, followed by macular and petechial $(6 / 42,15 \%)$ and macular $(4 / 42,10 \%)$. Involvement of palms and soles was common (30/38, $79 \%$ ) for those with non-ATBF rickettsiosis. Purpura fulminans was seen in 3 patients, all within the ISF group ( $9 \%$ of patients in this group). Eschar was present on $12 \%$ patients with ISF, $100 \%$ with ATBF, 1 of the 2 with $R$. conorii Malish infection, and 1 of the 3 
infected with the new Rickettsia variant. Except for 1 ISF patient who had 3 lesions, typically patients had only 1 eschar, usually on the lower limbs, including those in the ATBF group.

The time interval between fever onset and appearance of rash differed among the groups: for ISF patients, the mean (range) was $3.2(0-8)$ days, and for patients infected with the new Rickettsia variant, the mean and median were 15 days. Of the 2 patients with $R$. conorii Malish strain infection, 1 had rash that reportedly appeared 1 day before fever onset.

Systemic symptoms (e.g., myalgia and headache) were common for all patients with non-ATBF Rickettsia infection, and meningoencephalitis was evident in $8 / 33(24 \%)$ of patients with ISF. For ATBF patients, rates of systemic symptoms were lower. Similarly, about one third of patients with non-ATBF rickettsiosis but none with ATBF experienced severe disease with shock, multiorgan failure, need for mechanical ventilation, disseminated intravascular coagulation, acute respiratory distress syndrome, and need for intensive care.

\section{Mortality Rate and Related Risk Factors}

Of the 42 patients, 10 (23.8\%) died during their hospital stay, all within 20 days of admission (mean \pm SD [range] $6.3 \pm 6.3$ [1-20] days, median [IQR] was 3.5 [2.5-11] days). Mean age among those who died was 55 (14-95) years and among those who survived, 42.5
(1-83) years $(p=0.8)$. Mortality rate was remarkably high for the ISF group, reaching 30\%, although none in the other 3 groups died. The only patient-related risk factor significantly associated with death was alcohol abuse (hazard ratio [HR] 5.8, 95\% CI 1.14-30.4). Disease-related risk factors associated with death were hemodynamic shock at admission (HR 10.7, 95\% CI 1.33-87.3), disseminated intravascular coagulation (HR 4.7, 95\% CI 1.19-18.6), and jaundice (HR 6.7, 95\% CI 1.4-32.2). All patients in our study received doxycycline during hospitalization; the mean \pm SD (range) interval from fever onset to doxycycline receipt was 6 \pm 4.3 (1-29) days. This interval did not differ between groups of patients who survived (5.7 \pm 4.8 days) or died $(6.9 \pm 1.7$ days $)(p=0.4)$.

\section{Laboratory Data}

Except for ATBF, laboratory findings did not differ between the groups (Table 4). During the first 3 days of hospitalization, acute kidney injury was common $(>50 \%)$; other common findings included hepatic injury accompanied with mild to moderate jaundice, mild rhabdomyolysis, mild international normalized ratio prolongation, thrombocytopenia, and lymphocytopenia. Leukocytosis was more common than leukopenia, and C-reactive protein levels were $>100 \mathrm{mg} / \mathrm{L}$ (reference $<5 \mathrm{mg} / \mathrm{L}$ ). ATBF cases were distinctly different and patients showed much milder systemic reactions: no hepatocellular injury,

\begin{tabular}{|c|c|c|c|c|c|c|}
\hline & R. conorii Israeli tick & R. conorii & & New Rickettsia & & \\
\hline Patient data & typhus strain & Malish strain & $R$. africae & variant & Total & $p$ value \\
\hline No. cases & 33 & 2 & 4 & 3 & 42 & \\
\hline Age, y, median (IQR) & $48(18-64)$ & 38 & $55.5(52.5-64)$ & 66 & $50.5(26-66)$ & NS \\
\hline \multicolumn{7}{|l|}{ Sex } \\
\hline M & $20(61)$ & $1(50)$ & $2(50)$ & $2(67)$ & $25(60)$ & NS \\
\hline $\mathrm{F}$ & $13(39)$ & $1(50)$ & $2(50)$ & $1(33)$ & $17(40)$ & NS \\
\hline Any previous medical condition $\dagger$ & $17(51)$ & $1(50)$ & $1(25)$ & $2(66)$ & $21(50)$ & NS \\
\hline Diabetes mellitus & $6(18)$ & $1(50)$ & 0 & $1(33)$ & $8(19)$ & NS \\
\hline Dyslipidemia & $7(21)$ & 0 & 0 & $1(33)$ & $8(19)$ & NS \\
\hline Hypertension & $7(21)$ & 0 & 0 & 0 & $7(17)$ & NS \\
\hline Obesity & $3(9)$ & 0 & 0 & 0 & $3(7)^{\prime}$ & NS \\
\hline COPD & $1(3)$ & 0 & $1(25)$ & $1(33)$ & $3(7)$ & NS \\
\hline Chronic liver disease & $1(3)$ & $1(50)$ & 0 & 0 & $2(5)$ & NS \\
\hline Alcohol abuse & $3(9)$ & 0 & 0 & 0 & $3(7)$ & NS \\
\hline Psychiatric disorder/dementia & $3(9)$ & 0 & 0 & 0 & $3(7)$ & NS \\
\hline Drug abuse & $2(6)$ & 0 & 0 & 0 & $2(5)$ & NS \\
\hline Congestive heart failure & $2(6)$ & 0 & 0 & 0 & $2(5)$ & NS \\
\hline Chronic renal failure & $1(3)$ & 0 & 0 & 0 & $1(2.5)$ & NS \\
\hline \multicolumn{7}{|l|}{ Exposure history } \\
\hline Tick & $2(6)$ & 0 & 0 & 0 & $2(5)$ & NS \\
\hline Animals, species & $\begin{array}{c}20(60), \\
17 \text { dogs, } 4 \text { cats, } 2 \\
\text { rats, } 2 \text { sheep, } 1 \text { cow) }\end{array}$ & 0 & $\begin{array}{c}2(50), 1 \text { dog, } 1 \\
\text { African safari }\end{array}$ & $\begin{array}{c}3(100), \\
2 \text { dogs, } 1 \text { cat }\end{array}$ & $25(59)$ & NS \\
\hline Recent overseas travel & $1(3)$, to Cyprus & $1(50)$, to India & 4 (100), to Africa & 0 & $6(14)$ & $<0.0001$ \\
\hline
\end{tabular}


Table 3. Clinical features of patients with spotted fever group rickettsiosis, according to rickettsial species, Israel, 2010-2019*

\begin{tabular}{|c|c|c|c|c|c|c|}
\hline Patient data & $\begin{array}{l}\text { R. conorii Israeli } \\
\text { tick typhus strain }\end{array}$ & $\begin{array}{c}\text { R. conorii } \\
\text { Malish strain }\end{array}$ & R. africae & $\begin{array}{c}\text { New Rickettsia } \\
\text { variant }\end{array}$ & Total & $p$ value \\
\hline No. cases & 33 & 2 & 4 & 3 & 42 & \\
\hline Fever & $32(97)$ & $2(100)$ & $4(100)$ & $3(100)$ & $41(98)$ & NS \\
\hline $\begin{array}{l}\text { Fever to admission interval, } d, \text { mean } \\
( \pm S D \text {, range })\end{array}$ & $\begin{array}{c}4.5 \\
( \pm 2.1,0-8)\end{array}$ & $\begin{array}{c}6.5 \\
( \pm 3.5,4-9)\end{array}$ & $\begin{array}{c}3.5 \\
( \pm 2.4,1-6)\end{array}$ & $\begin{array}{c}13.6 \\
( \pm 13.3,5-29)\end{array}$ & $\begin{aligned} & 5.2 \\
&( \pm 4.3,0-29)\end{aligned}$ & $0.002 \dagger$ \\
\hline Systemic rash & $33(100)$ & $1(50)$ & 0 & $3(100)$ & $37(88)$ & $<0.0001 \ddagger$ \\
\hline \multicolumn{7}{|l|}{ Rash type } \\
\hline Macular only & $4(12)$ & 0 & 0 & 0 & $4(10)$ & \\
\hline Maculopapular & $19(58)$ & $1(100)$ & 0 & $2(67)$ & $22(53)$ & \\
\hline Macular and petechial & $5(15)$ & 0 & 0 & $1(33)$ & $6(15)$ & \\
\hline Petechial only & $2(6)$ & 0 & 0 & 0 & $2(5)$ & \\
\hline Purpura fulminans & $3(9)$ & 0 & 0 & 0 & $3(7)$ & \\
\hline \multicolumn{7}{|l|}{ Palm and sole involvement } \\
\hline Yes & $27(82)$ & $1(100)$ & 0 & $2(67)$ & $30(73)$ & $<0.0001 \ddagger$ \\
\hline No & $4(12)$ & 0 & $4(100)$ & $1(33)$ & $9(22)$ & \\
\hline Unknown & $2(6)$ & 0 & 0 & 0 & $2(5)$ & \\
\hline $\begin{array}{l}\text { Fever to rash interval, d, mean }( \pm S D \text {, } \\
\text { range }) \S\end{array}$ & $\begin{array}{c}3.2 \\
( \pm 2.2,0-8) \\
\end{array}$ & $-1 \rrbracket$ & NR & $\begin{array}{c}15 \\
( \pm 16.9,3-27) \#\end{array}$ & $\begin{array}{c}3.7 \\
( \pm 4.6,0-27) \\
\end{array}$ & \\
\hline Eschar & $4(12)$ & $1(50)$ & $4(100)$ & $1(33)$ & $10(24)$ & $0.011 \ddagger$ \\
\hline$>1$ eschar & $\begin{array}{l}1 \text { case }(3 \\
\text { lesions) }\end{array}$ & None & None & None & None & \\
\hline \multicolumn{7}{|l|}{ Location } \\
\hline Lower limb & 3 & 1 & 4 & 0 & 8 & \\
\hline Upper limb & 1 & 0 & 0 & 0 & 1 & \\
\hline Neck & 0 & 0 & 0 & 1 & 1 & \\
\hline \multicolumn{7}{|l|}{ Other signs/symptoms } \\
\hline Lymphadenitis & $2(6)$ & $1(50)$ & $1(25)$ & $1(33)$ & $5(12)$ & NS \\
\hline Lymphangitis & 0 & 0 & 0 & 0 & 0 & NS \\
\hline Myalgia & $13(39)$ & $2(100)$ & $1(25)$ & $2(67)$ & $18(43)$ & NS \\
\hline Arthralgia & $5(15)$ & 0 & 0 & $1(33)$ & $6(14)$ & NS \\
\hline Cough & $7(21)$ & $1(50)$ & 0 & $1(33)$ & $9(21)$ & NS \\
\hline Diarrhea & $7(21)$ & $2(100)$ & 0 & $1(33)$ & $10(24)$ & NS \\
\hline Rigors & $12(36)$ & $2(100)$ & $1(25)$ & 0 & $15(36)$ & NS \\
\hline Headache & $14(42)$ & $2(100)$ & $1(25)$ & $1(33)$ & $18(43)$ & NS \\
\hline Photophobia & $1(3)$ & 0 & 0 & 0 & $1(2)$ & NS \\
\hline Confusion & $10(30)$ & 0 & 0 & $2(67)$ & $12(28)$ & NS \\
\hline Meningoencephalitis & $8(24)$ & 0 & 0 & 0 & 8 (19) & NS \\
\hline Fever of unknown origin & $5(15)$ & 0 & 0 & $1(33)$ & $6(14)$ & NS \\
\hline ARDS & $10(30)$ & $1(50)$ & 0 & $2(67)$ & $13(31)$ & NS \\
\hline DIC & $10(30)$ & $1(50)$ & 0 & 0 & $11(26)$ & NS \\
\hline Shock & $13(39)$ & $2(100)$ & 0 & $1(33)$ & $16(38)$ & NS \\
\hline Hospitalization & $33(100)$ & $2(100)$ & $3(75)$ & $3(100)$ & $41(98)$ & NS \\
\hline LOS, d, mean ( \pm SD, range) & $\begin{array}{c}8.9 \\
( \pm 10.4,1-47)\end{array}$ & $( \pm 2.8,10-14)$ & $\begin{aligned} & 2.2 \\
&( \pm 2,0-4)\end{aligned}$ & $\begin{array}{c}57.6 \\
( \pm 91,5-163)\end{array}$ & $\begin{array}{c}11.9 \\
( \pm 25.6,0-163)\end{array}$ & $0.01 \dagger$ \\
\hline Intensive care admission & $17(52)$ & $2(100)$ & 0 & $1(33)$ & $20(48)$ & NS \\
\hline Mechanical ventilation & $13(39)$ & $2(100)$ & 0 & $1(33)$ & $16(38)$ & NS \\
\hline Death from rickettsiosis & $10(30)$ & 0 & 0 & 0 & $10(24)$ & $N S^{* *}$ \\
\hline \multicolumn{7}{|c|}{ 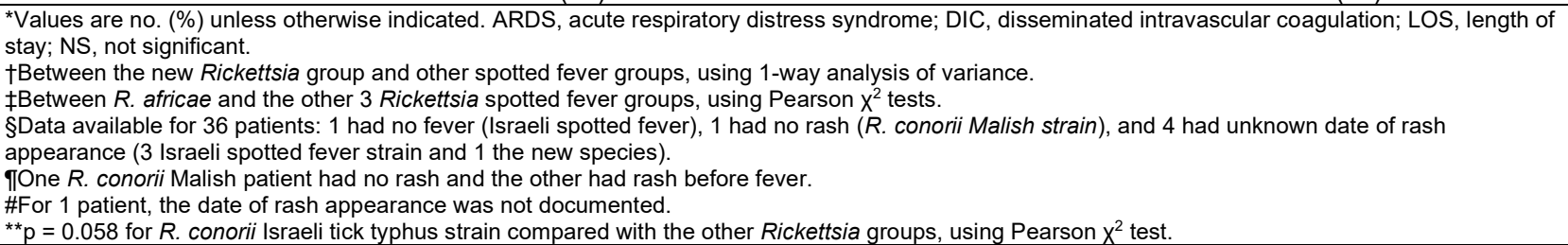 } \\
\hline
\end{tabular}

jaundice, rhabdomyolysis, or thrombocytopenia; and significantly lower C-reactive protein levels (mean 35 $\mathrm{mg} / \mathrm{L} ; \mathrm{p}=0.034)($ Table 4$)$.

\section{New Rickettsia Variant}

In 3 epidemiologically unrelated patients, we identified a new Rickettsia variant of the ISF clade. Partial sequencing of the following conserved Rickettsia genes indicated that the isolates were $100 \%$ identical to each other: gltA (GenBank accession no. MW366541), rOmpA (MW366542), and sca4 (MW366543). Highest similarity (92.2\%-96.9\% homology) was seen with $R$. conorii Astrakhan, $R$. slovaca, R. sibirica, and R. conorii ITTS. Phylogenetic analysis could not assign the new variant to any existing strain, as previously described (27). The 3 cases were unrelated spatially or temporally (Appendix, 
https:/ / wwwnc.cdc.gov/EID/article/27/8/20-3661App1.pdf).

\section{Discussion}

With this nationwide clinical and molecular study, we provide molecular evidence that $R$. conorii ITTS is the most commonly identified strain of SFG Rickettsia among hospitalized patients in Israel. Although SFGR is a reportable disease in Israel, it is underreported; of the average of 81 cases diagnosed by serologic testing each year according to the IIBR laboratory data, an average of 27 cases are reported to the Ministry of Health each year (24) (Figure 1, panel A). Because we lack clinical information for many of these cases, such as

Table 4. Laboratory features of 42 patients with spotted fever group rickettsiosis according to rickettsial species, during hospitalization days 1-3, Israel, 2010-2019*

\begin{tabular}{|c|c|c|c|c|c|c|}
\hline Feature $†$ & $\begin{array}{c}\text { R. conorii } \\
\text { Israeli tick } \\
\text { typhus strain }\end{array}$ & $\begin{array}{l}\text { R. conorii } \\
\text { Malish strain }\end{array}$ & R. africae & $\begin{array}{l}\text { New Rickettsia } \\
\text { variant }\end{array}$ & Total & $p$ value \\
\hline No. cases & 33 & 2 & 4 & 3 & 42 & \\
\hline $\begin{array}{l}\text { Acute kidney injury (creatinine } \geq 1.3 \\
\mathrm{mg} / \mathrm{dL} \text { ), no. }(\%)\end{array}$ & $20 / 32(62.5)$ & $0 / 2$ & $1 / 4(25)$ & $1 / 3(33)$ & $22 / 41(54)$ & NS $\ddagger$ \\
\hline Creatinine, mg/dL, mean (range) & $1.8(0.13-6.25)$ & $0.83(0.7-0.9)$ & $0.96(0.6-1.3)$ & $1.6(1.17-2.4)$ & $1.6(0.1-6.25)$ & NS§ \\
\hline $\begin{array}{l}\text { Hepatocellular injury pattern } \\
\text { AST or ALT >2 ULN, no. (\%) } \\
\text { AST, IU/L, mean (range) } \\
\text { ALT, IU/L, mean (range) }\end{array}$ & $\begin{array}{c}24 / 33(73) \\
855(42-8,895) \\
334(12-2,881) \\
\end{array}$ & $\begin{array}{c}2 / 2(100) \\
249(270-228) \\
94(109-80) \\
\end{array}$ & $\begin{array}{c}0 / 4(100) \\
27(23-31) \\
26(25-29) \\
\end{array}$ & $\begin{array}{c}2 / 3(67) \\
83(60-107) \\
88(40-164) \\
\end{array}$ & $\begin{array}{c}28 / 42(67) \\
728(23-8,895) \\
22 / 41(54) \\
\end{array}$ & $\begin{array}{l}0.02 \ddagger \\
\text { NS§ } \\
\text { NS§ }\end{array}$ \\
\hline \multicolumn{7}{|l|}{ Cholestatic injury pattern } \\
\hline $\begin{array}{l}\text { Alkaline phosphatase } \\
\text { or GGT >2 ULN, no. (\%) }\end{array}$ & $16 / 32(48)$ & $2 / 2(100)$ & $1 / 4(25)$ & $2 / 3(67)$ & $21 / 41(51)$ & NS $\ddagger$ \\
\hline $\begin{array}{l}\text { Alkaline phosphatase, IU/L, } \\
\text { mean (range) }\end{array}$ & $196(32-1,056)$ & $109(67-152)$ & $64(63-65)$ & $265(60-416)$ & $190(32-1,056)$ & NS§ \\
\hline GGT, IU/L, mean (range) & $156(15-1,026)$ & $150(129-171)$ & $73(21-125)$ & 507 & $154(15-1,026)$ & NS§ \\
\hline Jaundice, bilirubin $>1.3 \mathrm{mg} / \mathrm{dL}$, no. (\%) & $14 / 33(42)$ & $1 / 2(50)$ & $0 / 3$ & $0 / 3$ & $15 / 41(36)$ & NS $\ddagger$ \\
\hline Bilirubin, mg/dL, mean (range) & $1.77(0.29-10)$ & $2.6(1.3-4)$ & $0.4(0.3-0.5)$ & $0.93(0.6-1.3)$ & $1.46(0.29-10)$ & NS§ \\
\hline C-reactive protein $>5 \mathrm{mg} / \mathrm{L}$, no. $(\%)$ & $30 / 30(100)$ & $1 / 1(100)^{\prime}$ & $4 / 4(100)$ & $3 / 3(100)$ & $38 / 38(100)$ & NS $\ddagger$ \\
\hline C-reactive protein, $\mathrm{mg} / \mathrm{L}$, (range) & $207(17-460)$ & 102 & $35(17-61)$ & $223(131-273)$ & $187(17-410)$ & $0.034 \S$ \\
\hline $\begin{array}{l}\text { Rhabdomyolysis, creatine kinase } \\
>\text { ULN, no. }(\%)\end{array}$ & $17 / 30(52)$ & $1 / 2(50)$ & $0 / 2$ & $0 / 3$ & $18 / 37(49)$ & NS $\ddagger$ \\
\hline Creatine kinase, IU/L (range) & $\begin{array}{c}1,345 \\
(81-8,900)\end{array}$ & $\begin{array}{c}271 \\
(128-414)\end{array}$ & $\begin{array}{c}92 \\
(71-113)\end{array}$ & $\begin{array}{c}79 \\
(57-102)\end{array}$ & $\begin{array}{c}1,119 \\
(57-8,900)\end{array}$ & NS§ \\
\hline \multicolumn{7}{|l|}{ Complete blood count } \\
\hline $\begin{array}{l}\text { Leukocytosis, }>10,000 \\
\text { leukocytes/ } \mu \mathrm{L}, \text { no. (\%) }\end{array}$ & $15 / 33(45)$ & $0 / 2$ & $0 / 4$ & $1 / 3(33)$ & $16 / 42(38)$ & NS $\ddagger$ \\
\hline $\begin{array}{l}\text { Leukopenia, }<4,000 \\
\text { leukocytes } / \mu \mathrm{L}, \text { no. }(\%)\end{array}$ & $6 / 33(18)$ & $1 / 2(50)$ & $0 / 4$ & $1 / 3(33)$ & $8 / 42(19)$ & NS $\ddagger$ \\
\hline Leukocytes, $\times 10^{3} / \mu \mathrm{L}$, mean (range) & $14.2(2.5-43.3)$ & $4.5(2.6,6.4)$ & $4.5(4-5.2)$ & $10.2(3.9-17.1)$ & $13.1(2.5-43.3)$ & NS§ \\
\hline 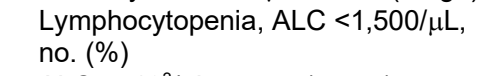 & $30 / 33(91)$ & $2 / 2(100)$ & $4 / 4(100)$ & $3 / 3(100)$ & $36 / 42(93)$ & NS $\ddagger$ \\
\hline $\mathrm{ALC}, \times 10^{3} / \mu \mathrm{L}$, mean (range) & $0.9(0.2-6.9)$ & $0.35(0.3-0.4)$ & $1.26(1.2-1.3)$ & $1.1(0.6-2.3)$ & $0.9(0.2-6.9)$ & NS§ \\
\hline $\begin{array}{l}\text { Thrombocytopenia, platelets }<150 \mathrm{~K} \\
\text { cells } / \mu \mathrm{L} \text {, no. }(\%)\end{array}$ & $29 / 32(88)$ & $2 / 2(100)$ & $0 / 4$ & $3 / 3(100)$ & $34 / 41(83)$ & $0.001 \ddagger$ \\
\hline Platelets, $\times 10^{3} / \mu \mathrm{L}$, mean (range) & $\begin{array}{c}84 \\
(15-271) \\
\end{array}$ & $\begin{array}{c}36 \\
(26-46) \\
\end{array}$ & $\begin{array}{c}238 \\
(164-316)\end{array}$ & $\begin{array}{c}64 \\
(37-101)\end{array}$ & $\begin{array}{c}82 \\
(15-271)\end{array}$ & $0.001 \S$ \\
\hline Coagulopathy, INR >1.2, no. (\%) & $16 / 33(48)$ & $1 / 2(50)$ & $0 / 2$ & $0 / 3$ & $17 / 40(42)$ & NS $\ddagger$ \\
\hline INR, mean (range) & $\begin{array}{c}1.38 \\
(0.9-3)\end{array}$ & $\begin{array}{c}1.5 \\
(1.1-1.9)\end{array}$ & $\begin{array}{c}0.96 \\
(0.93-1)\end{array}$ & $\begin{array}{c}1.09 \\
(1.04-1.16)\end{array}$ & $1.36(0.9-3)$ & NS§ \\
\hline \multicolumn{7}{|l|}{ Molecular diagnosis source, no. (\%) } \\
\hline Skin biopsy sample/eschar & $19(58)$ & $1(50)$ & $4(100)$ & $3(100)$ & $27(64)$ & \\
\hline Blood & $16(48)$ & $2(100)$ & 0 & $1(33)$ & $19(45)$ & \\
\hline CSF & $1(3)$ & 0 & 0 & 0 & $1(2)$ & \\
\hline Other organs & $2(6) \pi$ & 0 & 0 & 0 & $2(5)$ & \\
\hline Serologic diagnosis, no. (\%) samples & $32(97)$ & $2(100)$ & $1(25)$ & $1(33)$ & $36(86)$ & \\
\hline Positive & $5(16)$ & 0 & 0 & 0 & $5(14)$ & \\
\hline Borderline & $2(6)$ & 0 & 0 & $1(33)$ & $3(8)$ & \\
\hline Negative & $25(78)$ & $2(100)$ & $1(25)$ & 0 & $28(78)$ & \\
\hline
\end{tabular}


severity of illness and hospitalization, we can only partially infer the role of this strain in causing SFGR among hospitalized and ambulatory patients. Although $R$. conorii ITTS was suspected as the causative agent of SFGR in Israel, this suspicion has been supported only by very limited data: rare case reports of fatal human cases $(9,15)$ and a few studies of ticks $(25,32)$.

Testing for spotted fever by serology and recently by PCR increased during 2017-2019 compared with the preceding 3 years. This rise probably represents increased clinical suspicion of SFGR and a true increase in disease activity. Despite missing molecular data for ambulatory patients with mild cases of SFGR, we believe that during the past 3 years, $R$. conorii ITTS has led a silent outbreak of SFGR in Israel. Similarly, an unprecedented $46 \%$ increase in SFGR was reported during 2016-2017 by the US Centers for Disease Control and Prevention from states where SFGR is known to be endemic (33). US authorities were faced with the dilemma of whether this increased incidence should be ascribed to a true increase, increased testing, or inappropriate use of a single serologic test instead of paired tests, reflecting past infection in a disease-endemic area.

We report the epidemiologic features of SFGR patients, although comparison between $R$. conorii ITTS with the other groups was limited because of small numbers. In Israel, SFGR affects mainly young adults and has a mild predilection for men. SFGR has been endemic to the Sharon and Haifa Districts since the 1990s (34); in our study, these 2 districts accounted for $44 \%(16 / 36)$ of all autochthonous cases. These results are similar to those reported in 2014 by Rose et al., who also investigated the association between geographic data and SFGR-positive ticks. Areas with SFG Rickettsia-infected ticks were associated with browntype soil, higher land surface temperatures, and higher precipitation (31). We observed a wide geographic distribution of human ISF cases with aggregation in northern Israel.

Although $R$. conorii ITTS seems to be the main Rickettsia causing clinical disease in Israel, why this strain is rarely found in ticks collected from Israel $(25,35)$ and other countries $(7,36,37)$ remains unclear. Studies from Israel have found Rickettsia massiliae to be more prevalent $(\approx 10$ fold) than $R$. conorii ITTS among questing ticks and ticks feeding on animals $(31,35,38)$. This discrepancy could be explained by underreporting of R. massiliae infection in humans with mild or subclinical disease. Most patients in our study were hospitalized with severe disease and may represent a reporting bias of $R$. conorii ITTS, which causes more severe disease. Patients with milder illness, potentially caused by other rickettsiae, may not be hospitalized, and illness may resolve undiagnosed, without the need for molecular studies.

Rose et al. (31) collected Rhipicephalus sanguineus and Rh. turanicus ticks from geographic locations similar to the presumed areas of the clinical autochthonous cases in our study (Figure 2, panel B). However, R. conorii ITTS was found only rarely $(1.8 \%)$ and strictly in $R h$. sanguineus ticks. Hence, the role of $R h$. turanicus ticks as possible vectors of $R$. conorii ITTS and $R$. massiliae as a cause of rickettsiosis in humans should be further explored. Although clearly reported as a cause of SFGR, $R$. massiliae is still rarely isolated from human patients (39). Deleterious effects of $R$. conorii on $R h$. sanguineus tick fitness, resulting in infected ticks not surviving the winter, may explain its low prevalence among ticks in nature $(40,41)$.

The new Rickettsia variant was found in the eastern and more mountainous parts of the country: the Golan Heights, the Galilee region, and the West Bank of the Palestinian Authority. This distribution may suggest a geographic niche for either this new Rickettsia or its vector and should be further explored in studies of tick collections from these mountainous areas. The single case of $R$. conorii Malish strain infection acquired locally in this study was in a 50-year-old man from the desert area, who had a necrotic eschar in the thigh and severe systemic disease.

Most cases were reported during the summer and peaked in August. This finding is consistent with previous reports (12) and may be attributed to increased activity of the vectors and to the aggressiveness and host indiscrimination of $R h$. sanguineus ticks when exposed to higher temperatures (42).

Patients rarely remembered seeing or being bitten by ticks (only 2 remembered); however, exposure to animals was common (25/42, 59\% of cases), mainly to dogs $(20 / 25,80 \%)$, the principal hosts of $R h$. sanguineus ticks (the main reservoir of $R$. conorii). This finding implies that exposure to domestic pets is more relevant than exposure to ticks.

The clinical and laboratory features for patients in our case series were typical of SFGR, although eschar, which is considered rare in patients with ISF, was seen in $12 \%$ of patients, 1 of whom had 3 lesions. ISF caused purpura fulminans in $9 \%$ and meningoencephalitis in $24 \%$. About half of ISF patients experienced multiorgan involvement that included kidney and liver injury, jaundice, rhabdomyolysis/myositis, and coagulopathy. Severe disease requiring intensive care was strikingly common (52\%), and $30 \%$ of ISF patients died in hospital. The high mortality rate, 
previously reported for ISF infection (10), contrasted with lower rates from historical reports from Israel during the 1990s. This discrepancy may result from reporting bias with increased awareness in recent years, as well as improved laboratory capabilities. An additional possibility is an outbreak of a more virulent strain, such as $R$. conorii ITTS. Risk factors for death included only alcohol abuse, as previously described (12). Admission-to-treatment (doxycycline) interval was not significant.

The small number of cases in this investigation makes drawing conclusions or comparisons difficult; however, the new Rickettsia variant may lead to prolonged fever before care seeking and may resemble tickborne lymphadenopathy usually related to Rickettsia slovaca or Rickettsia raoultii (11). Patients with ATBF had a distinct clinical syndrome of a milder clinical disease; for $25 \%$, systemic symptoms were limited to fever, myalgia, and headache with no systemic rash.

In conclusion, we report a nationwide case series of hospitalized patients with molecularly diagnosed SFGR over a decade in Israel, of which $R$. conorii ITTS was the principal cause of severe disease, multiorgan failure, and high mortality rates. We also describe a new Rickettsia variant, which may be associated with unique epidemiologic and clinical features. This study suggests that a broader range of species causes SFGR in Israel and that this possibility should be explored in larger, prospective studies, especially in light of the potential candidates found in ticks.

\section{Acknowledgments}

We thank Shimon Harrus for his permission to adapt the data from the study of SFG Rickettsia in questing ticks in Israel.

\section{About the Author}

Dr. Cohen is the head of the infectious diseases unit and infection control unit in Sanz Medical Center, Laniado Hospital, Netanya, Israel. His research interests are zoonoses and healthcare-associated infections, including resistant microorganisms and viral respiratory infections.

\section{References}

1. Diop A, Raoult D, Fournier PE. Rickettsial genomics and the paradigm of genome reduction associated with increased virulence. Microbes Infect. 2018;20:401-9. https:/ / doi.org/ 10.1016/j.micinf.2017.11.009

2. Blanton LS, Walker DH. Rickettsia rickettsii and other spotted fever group rickettsiae (Rocky Mountain spotted fever and other spotted fevers). In: Bennett JE, Dolin R, Blaser MJ, editors. Mandell, Douglas, and Bennett's principles and practice of infectious diseases, 9th ed. Amsterdam: Elsevier; 2020.
3. Parola P, Paddock CD, Socolovschi C, Labruna MB, Mediannikov O, Kernif T, et al. Update on tick-borne rickettsioses around the world: a geographic approach. Clin Microbiol Rev. 2013;26:657-702. https://doi.org/10.1128/ CMR.00032-13

4. Bacellar F, Beati L, França A, Poças J, Regnery R, Filipe A. Israeli spotted fever rickettsia (Rickettsia conorii complex) associated with human disease in Portugal. Emerg Infect Dis. 1999;5:835-6. https://doi.org/10.3201/eid0506.990620

5. Colomba C, Trizzino M, Giammanco A, Bonura C, Di Bona D, Tolomeo M, et al. Israeli spotted fever in Sicily. Description of two cases and minireview. Int J Infect Dis. 2017;61:7-12. https://doi.org/10.1016/j.ijid.2017.04.003

6. Zhu Y, Fournier PE, Eremeeva M, Raoult D. Proposal to create subspecies of Rickettsia conorii based on multi-locus sequence typing and an emended description of Rickettsia conorii. BMC Microbiol. 2005;5:11. https:/ / doi.org/10.1186/ 1471-2180-5-11

7. Znazen A, Hammami B, Lahiani D, Ben Jemaa M, Hammami A. Israeli spotted fever, Tunisia. Emerg Infect Dis. 2011;17:1328-30. https://doi.org/10.3201/eid1707.101648

8. Angelakis E, Richet H, Raoult D. Rickettsia sibirica mongolitimonae infection, France, 2010-2014. Emerg Infect Dis. 2016;22:880-2. https:// doi.org/10.3201/eid2205.141989

9. Cohen R, Babushkin F, Shapiro M, Uda M, Atiya-Nasagi Y, Klein D, et al. Two cases of Israeli spotted fever with purpura fulminans, Sharon District, Israel. Emerg Infect Dis. 2018;24:835-40. https://doi.org/10.3201/eid2405.171992

10. de Sousa R, Nóbrega SD, Bacellar F, Torgal J. Mediterranean spotted fever in Portugal: risk factors for fatal outcome in 105 hospitalized patients. Ann N Y Acad Sci. 2003;990:285-94. https:/ / doi.org/10.1111/j.1749-6632.2003.tb07378.x

11. Parola P, Rovery C, Rolain JM, Brouqui P, Davoust B, Raoult D. Rickettsia slovaca and $R$. raoultii in tick-borne rickettsioses. Emerg Infect Dis. 2009;15:1105-8. https:// doi.org/10.3201/eid1507.081449

12. Sousa R, França A, Dória Nòbrega S, Belo A, Amaro M, Abreu T, et al. Host- and microbe-related risk factors for and pathophysiology of fatal Rickettsia conorii infection in Portuguese patients. J Infect Dis. 2008;198:576-85. https://doi.org/10.1086/590211

13. Raoult D, Weiller PJ, Chagnon A, Chaudet H, Gallais H, Casanova P. Mediterranean spotted fever: clinical, laboratory and epidemiological features of 199 cases. Am J Trop Med Hyg. 1986;35:845-50. https:/ / doi.org/10.4269/ ajtmh.1986.35.845

14. Aharonowitz G, Koton S, Segal S, Anis E, Green MS. Epidemiological characteristics of spotted fever in Israel over 26 years. Clin Infect Dis. 1999;29:1321-2. https:/ / doi.org/ $10.1086 / 313432$

15. Weinberger M, Keysary A, Sandbank J, Zaidenstein R, Itzhaki A, Strenger C, et al. Fatal Rickettsia conorii subsp. israelensis infection, Israel. Emerg Infect Dis. 2008;14:821-4. https://doi.org/10.3201/eid1405.071278

16. Paddock CD, Childs JE, Zaki SR, Berger SA. Reply. J Infect Dis. 2000;181:810-2. https://doi.org/10.1086/315288

17. Leitner M, Yitzhaki S, Rzotkiewicz S, Keysary A. Polymerase chain reaction-based diagnosis of Mediterranean spotted fever in serum and tissue samples. Am J Trop Med Hyg. 2002;67:166-9. https://doi.org/10.4269/ajtmh.2002.67.166

18. Regev-Yochay G, Segal E, Rubinstein E. Glucose-6-phosphate dehydrogenase deficiency: possible determinant for a fulminant course of Israeli spotted fever. Isr Med Assoc J. 2000;2:781-2.

19. Ergas D, Sthoeger ZM, Keysary A, Strenger C, Leitner M, Zimhony O. Early diagnosis of severe Mediterranean spotted 
fever cases by nested-PCR detecting spotted fever rickettsiae $17-k D$ common antigen gene. Scand J Infect Dis. 2008; 40:965-7. https:// doi.org/10.1080/00365540802400584

20. Cohen J, Lasri Y, Landau Z. Mediterranean spotted fever in pregnancy. Scand J Infect Dis. 1999;31:202-3. https:/ / doi.org/ 10.1080/003655499750006290

21. Bentov Y, Sheiner E, Kenigsberg S, Mazor M. Mediterranean spotted fever during pregnancy: case presentation and literature review. Eur J Obstet Gynecol Reprod Biol. 2003;107:214-6. https:// doi.org/10.1016/S0301-2115 (02)00303-2

22. Shazberg G, Moise J, Terespolsky N, Hurvitz H. Family outbreak of Rickettsia conorii infection. Emerg Infect Dis. 1999;5:723-4. https:// doi.org/10.3201/eid0505.990518

23. Cwikel BJ, Ighbarieh J, Sarov I. Antigenic polypeptides of Israeli spotted fever isolates compared with other spotted fever group rickettsiae. Ann N Y Acad Sci. 1990;590(1 Rickettsiolog):381-8. https:// doi.org/10.1111/ j.1749-6632. 1990.tb42244.x

24. Israel Center for Disease Control Ministry of Health. Publication files, infectious diseases, notifiable infectious diseases in Israel [in Hebrew] [cited 2020 Apr 15]. https://www.health.gov.il/UnitsOffice/HD/PH/ epidemiology/Pages/epidemiology_report.aspx

25. Harrus S, Perlman-Avrahami A, Mumcuoglu KY, Morick D, Baneth G. Molecular detection of Rickettsia massiliae, Rickettsia sibirica mongolitimonae and Rickettsia conorii israelensis in ticks from Israel. Clin Microbiol Infect. 2011;17:176-80. https://doi.org/10.1111/j.1469-0691.2010.03224.x

26. Keysary A, Strenger C. Use of enzyme-linked immunosorbent assay techniques with cross-reacting human sera in diagnosis of murine typhus and spotted fever. J Clin Microbiol. 1997;35:1034-5. https:// doi.org/10.1128/ JCM.35.4.1034-1035.1997

27. Klein D, Beth-Din A, Cohen R, Lazar S, Glinert I, Zayyad H, et al. New spotted fever group Rickettsia isolate, identified by sequence analysis of conserved genomic regions. Pathogens. 2019;9:E11. https:/ / doi.org/10.3390/ pathogens9010011

28. Roux V, Raoult D. Phylogenetic analysis of members of the genus Rickettsia using the gene encoding the outer-membrane protein rOmpB $(o m p B)$. Int J Syst Evol Microbiol. 2000;50:1449-55. https:/ / doi.org/10.1099/ 00207713-50-4-1449

29. Sekeyova Z, Roux V, Raoult D. Phylogeny of Rickettsia spp. inferred by comparing sequences of 'gene $\mathrm{D}^{\prime}$, which encodes an intracytoplasmic protein. Int J Syst Evol Microbiol. 2001;51:1353-60. https:/ / doi.org/10.1099/ 00207713-51-4-1353

30. Bechah Y, Socolovschi C, Raoult D. Identification of rickettsial infections by using cutaneous swab specimens and PCR. Emerg Infect Dis. 2011;17:83-6. https:/ / doi.org/ 10.3201/eid1701.100854

31. Rose J, Nachum-Biala Y, Mumcuoglu KY, Alkhamis MA, Ben-Nun A, Lensky I, et al. Genetic characterization of spotted fever group rickettsiae in questing ixodid ticks collected in Israel and environmental risk factors for their infection. Parasitology. 2017;144:1088-101. https://doi.org/ $10.1017 /$ S0031182017000336

32. Sentausa E, El Karkouri K, Robert C, Raoult D, Fournier PE. Genome sequence of Rickettsia conorii subsp. israelensis, the agent of Israeli spotted fever. J Bacteriol. 2012;194:5130-1. https://doi.org/10.1128/JB.01118-12

33. Heitman KN, Drexler NA, Cherry-Brown D, Peterson AE, Armstrong PA, Kersh GJ. National surveillance data show increase in spotted fever rickettsiosis: United States, 2016-2017. Am J Public Health. 2019;109:719-21. https://doi.org/10.2105/AJPH.2019.305038

34. Mumcuoglu KY, Keysary A, Gilead L. Mediterranean spotted fever in Israel: a tick-borne disease. Isr Med Assoc J. 2002;4:44-9.

35. Ereqat S, Nasereddin A, Al-Jawabreh A, Azmi K, Harrus S, Mumcuoglu K, et al. Molecular detection and identification of spotted fever group rickettsiae in ticks collected from the West Bank, Palestinian Territories. PLoS Negl Trop Dis. 2016;10:e0004348. https:// doi.org/10.1371/ journal.pntd.0004348

36. Kamani J, Baneth G, Mumcuoglu KY, Waziri NE, Eyal O, Guthmann Y, et al. Molecular detection and characterization of tick-borne pathogens in dogs and ticks from Nigeria. PLoS Negl Trop Dis. 2013;7:e2108. https:/ / doi.org/10.1371/ journal.pntd.0002108

37. Chisu V, Masala G, Foxi C, Socolovschi C, Raoult D, P arola P. Rickettsia conorii israelensis in Rhipicephalus sanguineus ticks, Sardinia, Italy. Ticks Tick Borne Dis. 2014;5:446-8. https:/ / doi.org/10.1016/j.ttbdis.2014.02.003

38. Waner T, Keysary A, Eremeeva ME, Din AB, Mumcuoglu KY, King R, et al. Rickettsia africae and Candidatus Rickettsia barbariae in ticks in Israel. Am J Trop Med Hyg. 2014;90:9202. https:// doi.org/10.4269/ajtmh.13-0697

39. Eldin C, Virgili G, Attard L, Edouard S, Viale P, Raoult D, et al. Rickettsia massiliae infection after a tick bite on the eyelid. Travel Med Infect Dis. 2018;26:66-8. https:/ / doi.org/ 10.1016/j.tmaid.2018.08.002

40. Socolovschi C, Gaudart J, Bitam I, Huynh TP, Raoult D, Parola P. Why are there so few Rickettsia conorii conorii-infected Rhipicephalus sanguineus ticks in the wild? PLoS Negl Trop Dis. 2012;6:e1697. https://doi.org/10.1371/journal.pntd.0001697

41. Parola P, Socolovschi C, Raoult D. Deciphering the relationships between Rickettsia conorii conorii and Rhipicephalus sanguineus in the ecology and epidemiology of Mediterranean spotted fever. Ann N Y Acad Sci. 2009; 1166:49-54. https:// doi.org/10.1111/j.1749-6632. 2009.04518.x

42. Socolovschi C, Raoult D, Parola P. Influence of temperature on the attachment of Rhipicephalus sanguineus ticks on rabbits. Clin Microbiol Infect. 2009;15(Suppl 2):326-7. https:/ / doi.org/ 10.1111/j.1469-0691.2008.02260.x

Address for correspondence: Regev Cohen, Head of Infectious Diseases Unit, Sanz Medical Center, 16 Divrei Haim St, Kiryat Sanz, 42150, Netanya, Israel; email: regevco@gmail.com 